\title{
Physical Conditions in NGC 6543
}

\section{Nancy Jo Lame ${ }^{1}$, J. Patrick Harrington ${ }^{1}$ and K. Borkowski ${ }^{2}$ \\ ${ }^{1}$ University of Maryland, College Park; ${ }^{2}$ North Carolina State University}

We observed NGC 6543 (the Cat's Eye Nebula) with the HST WFPC2 camera in ten narrow-band filters covering a range of emission lines. These images are used to further look at the physical conditions in NGC 6543. We discuss the [O III] electron temperature, ionization state throughout the nebula, and the results of photoionization models of individual structures.

The [O III] $\lambda 5007,4363$ emission-line images were used to make a full map of the [O III] electron temperature across NGC 6543. To reduce noise the [O III] images were binned and the temperature map smoothed, resulting in a final spatial resolution of about $0.2^{\prime \prime}$. The $\lambda 4363$ image was corrected for nebular continuum $(0.015 \mathrm{~F}(\mathrm{H} \beta))$ and $\mathrm{H} \gamma$ contamination by subtracting a scaled $\mathrm{H} \beta$ image.

The mean [O III] temperature is $8000 \mathrm{~K}$ with a standard deviation of $625 \mathrm{~K}$, measured from the peak and width of the histogram of temperatures. Although the map is noisy in a number of regions, in general large variations in temperature are not seen, with $90 \%$ of the pixels showing a temperature in the range $7000 \mathrm{~K}-9000 \mathrm{~K}$. No distinct temperature differences are seen in either the [O III]-bright "bubble" or the [O I]-bright equatorial ring. However, small temperature differences may exist between nebular components. The temperature appears to rise in the core and towards the central star, to over $10,000 \mathrm{~K}$, but temperature values in this region are uncertain because the central star is very saturated in the $\lambda 4363$ image. Any wings on the WFPC2 stellar PSF would have a significant effect on the temperature determination. The temperature appears to increase slightly in the caps, to $\sim 8300 \mathrm{~K}$ from $\sim 7700 \mathrm{~K}$ just interior to the caps. Although $\lambda 4363$ flux is very weak past the caps near the ansae, temperature seems to drop to $\sim 7200 \mathrm{~K}$ in this region. The $\lambda 4363$ flux is not high enough to measure temperature in the jets.

The variation seen in the [O III] electron temperature map is small - about $12 \%$. A typical value for the temperature fluctuation parameter $t^{2}$ is 0.04 , or a $20 \%$ variation in temperature (Kingdon \& Ferland 1995). Kingsburgh et al. (1996) measured $t^{2}=0.057$ in NGC 6543 using the forbidden and recombination lines of the $\mathrm{O}^{++}$ion. However, the temperature variations implied by either $t^{2}$ are not seen in our temperature map.

The ionization state in the nebula is illustrated with a false-color map made by combining the [O III] $\lambda 5007$, [O II] $\lambda 3727$, and [O I] $\lambda 6300$ images. As expected, the knots in the equatorial ring have neutral cores ([O I]) surrounded by ionized gas ([O II]). Similarly, the ansae have a filament of [O I] running through them which is surrounded by strong [O II] emission. The high-ionization gas ([O III]) is not confined to the elliptical bubble but extends out, disk-like, to the ring of knots.

The high spatial resolution of the WFPC2 images enables isolation of emission from specific, small-scale components of NGC 6543 such as the jets, ansae and caps. Isolated fluxes can be difficult to measure accurately with most ground-based telescopes and instruments with component dimensions as small as $1^{\prime \prime}$. Synthesized spectra from the ten emission lines observed are presented for each of the isolated structures in Table 1. The 


\section{Envelopes}

Table 1: Emission-line Fluxes ${ }^{a}$ of Isolated Components

\begin{tabular}{lcccccccc}
\hline \hline \multicolumn{1}{c}{ line } & $\lambda$ & global $^{b}$ & core & ring & caps $^{c}$ & ansae $^{c}$ & N jet & S jet \\
\hline $\mathrm{H} \beta$ & 4861 & 1.000 & 1.000 & 1.000 & 1.000 & 1.000 & 1.000 & 1.000 \\
$\mathrm{H} \alpha$ & 6563 & 2.992 & 2.913 & 3.066 & 3.024 & 2.960 & 3.322 & 3.666 \\
$\mathrm{HeI}$ & 5876 & 0.205 & 0.215 & 0.202 & 0.203 & 0.199 & 0.211 & 0.115 \\
{$[\mathrm{OIII}]$} & 5007 & 6.211 & 6.481 & 6.283 & 6.224 & 5.145 & 5.811 & 4.895 \\
{$[\mathrm{OIII}]$} & 4363 & 0.047 & 0.068 & 0.034 & 0.037 & 0.066 & 0.118 & 0.230 \\
{$[\mathrm{OII}]$} & 3727 & 0.420 & 0.268 & 0.308 & 0.881 & 2.042 & 4.715 & 3.095 \\
{$[\mathrm{OI}]$} & 6300 & 0.045 & 0.041 & 0.040 & 0.083 & 0.143 & 0.345 & 0.242 \\
{$[\mathrm{NII}]$} & 6584 & 0.345 & 0.050 & 0.260 & 1.302 & 2.411 & 4.258 & 3.608 \\
{$[\mathrm{SIII}]$} & 9531 & 1.047 & 0.730 & 1.172 & 1.380 & 1.129 & 1.091 & 1.009 \\
{$[\mathrm{SII}]$} & 6724 & 0.097 & 0.059 & 0.079 & 0.226 & 0.498 & 0.928 & 0.942 \\
\hline \hline
\end{tabular}

${ }^{a}$ fluxes are not corrected for nebular continuum or reddening

${ }^{b}$ sum in ellipse with major axis $=40.5^{\prime \prime}$, minor axis $=22.5^{\prime \prime}$

${ }^{c}$ total in point-symmetric structures

SYNPHOT package was used for flux calibration of the images. The fluxes are normalized to $\mathrm{H} \beta=1.0$, and are not corrected for nebular continuum $(\sim 2 \% \mathrm{H} \beta)$ or reddening. Although the $\mathrm{H} \alpha$, [N II], and [O III] $\lambda 4363$ fluxes are corrected for bandpass contamination by neighboring lines, the [O I] $\lambda 6300$ fluxes still contain a contribution from [S III] $\lambda 6312$ which is sizable in some apertures.

We present two photoionization models which show strong emission in the low-ionization lines of [O II], [N II], [S II] and [O I] similar to what we observe in the caps and ansae of NGC 6543. We do not claim the models are unique. The models were made using the photoionization code CLOUDY (Ferland 1993), with the central star spectrum model of de Koter et al. (1996, $\left.\mathrm{T}_{e f f}=48,000\right)$. The central star ionizing fluxes (de Koter, private communication) were scaled for a distance of $1.5 \mathrm{kpc}$. The abundances used for the models were close to average: $\mathrm{He} / \mathrm{H}=0.12, \mathrm{C} / \mathrm{H}=7.94 \mathrm{E}-4, \mathrm{~N} / \mathrm{H}=1.26 \mathrm{E}-4, \mathrm{O} / \mathrm{H}=5.01 \mathrm{E}$ $4, \mathrm{Ne} / \mathrm{H}=9.77 \mathrm{E}-5, \mathrm{~S} / \mathrm{H}=8.91 \mathrm{E}-6$. These models show that it is not necessary to resort to shock-heating or abundance anomalies to explain the spectrum of the caps and ansae in NGC 6543.

\section{REFERENCES}

de Koter, A., Heap, S.R., Hubeny, I., \& Lanz, T. 1996, preprint.

Ferland, G. J. 1993, University of Kentucky Department of Physics and Astronomy Internal

Report.

Kingdon, J.B., \& Ferland, G.J. 1995, ApJ, 450,691.

Kingsburgh, R. L., López, J. A., \& Peimbert, M. 1996, in: Cosmic Abundances, eds. S. Holt and G. Sonneborn, ASP Conf. Ser. 99, p. 350. 